\title{
Extended-State-Observer-Based Collision-Free Guidance Law for Target Tracking of Autonomous Surface Vehicles with Unknown Target Dynamics
}

\author{
Shengnan Gao $(\mathbb{D}$, Zhouhua Peng $(\mathbb{D}$, Dan Wang $(\mathbb{D}$, and Lu Liu \\ School of Marine Electrical Engineering, Dalian Maritime University, Dalian, China \\ Correspondence should be addressed to Zhouhua Peng; zhpeng@dlmu.edu.cn
}

Received 17 August 2018; Accepted 25 October 2018; Published 13 December 2018

Guest Editor: Junpei Zhong

Copyright (c) 2018 Shengnan Gao et al. This is an open access article distributed under the Creative Commons Attribution License, which permits unrestricted use, distribution, and reproduction in any medium, provided the original work is properly cited.

\begin{abstract}
This paper is concerned with the target tracking problem of an autonomous surface vehicle in the presence of a maneuvering target. The velocity information of target is totally unknown to the follower vehicle, and only the relative distance and angle between the target and follower are obtained. First, a reduced-order extended state observer is used to estimate the unknown relative dynamics due to the unavailable velocity of the target. Based on the reduced-order extended state observer, an antidisturbance guidance law for target tracking is designed. The input-to-state stability of the closed-loop target tracking guidance system is analyzed via cascade theory. Furthermore, the above result is extended to the case that collisions between the target and leader are avoided during tracking, and a collision-free target tracking guidance law is developed. The main feature of the proposed guidance law is twofold. First, the target tracking can be achieved without using the velocity information of the target. Second, collision avoidance can be achieved during target tracking. Simulation results show the effectiveness of the proposed antidisturbance guidance law for tracking a maneuvering target with the arbitrary bounded velocity.
\end{abstract}

\section{Introduction}

Recently, advanced motion control of marine vehicles has received significant attention due to its wide applications in hydrological monitoring, channel exclusion, search and rescue, biological detection, and so on [1-7]. Numerous motion control scenarios of marine vehicles have been considered including path following [8-21], path tracking [22, $23]$, trajectory tracking $[4,24-26]$, and target tracking $[2,27-$ 34]. Target tracking is to track a maneuvering target where no information about the target behavior is known in advance except its instantaneous motion.

Various control methods have been developed for the target tracking of marine vehicles [2, 27-34]. In [2], a position tracking controller is developed for an underactuated autonomous underwater vehicle based on a backstepping technique and neural networks. In [27], a straight-line target tracking controller is developed for an underactuated unmanned surface vehicle. In [28], a target tracking controller is developed for underactuated autonomous surface vehicles (ASVs) with limited control torques. In [29], a fault tolerant target tracking controller is developed for underactuated ASVs. In [2, 27-29], the velocities of the targets are known as a priori. In practice, however, the velocity information of the target may not be available by the follower. In order to track the leader in the absence of velocity information of target, a variety of methods are available [30, 31]. In [30], a robust controller is designed for target tracking of marine vessels where unknown velocity of the leader is handled by using a sliding model control. In [31], an adaptive leader-follower formation controller is designed for ASVs based on a dynamic surface control and singlehidden-layer neural networks, and an adaptive term is used to estimate the unknown velocity of target. In recent years, collision avoidance has been considered in motion control $[35,36]$. However, the collision avoidance problem during target tracking is not considered in [2, 27-29].

Extended state observer as a key part of active disturbance rejection control method was proposed by Han [37-39]. It is a powerful tool to deal with the nonlinear systems in 
the presence of large uncertainty including internal model uncertainties and external disturbances [40]. It has been widely used in numerous engineering applications [11, 41, 42]. A reduced-order extended state observer (RESO) is able to decrease the phase lag and simplify the observer structure to reduce computation load. It has the advantages of fast observation and no overshooting. Because of its advantages, it is desirable to employ the extended state observer to address the uncertainty during target tracking.

In this paper, an antidisturbance guidance law for target tracking is designed based on the reduced-order extended state observer (RESO), where an ASV is requested to track a maneuvering target. Only relative line-of-sight range and angle between the follower and target are available for feedback design. At first, a RESO is used to estimate the unknown relative dynamics due to the unavailable velocity of the target. Then, an antidisturbance guidance law is proposed based on the RESO and the stability of closed-loop guidance system is analyzed via cascade analysis. The above result is extended to target tracking with collision avoidance of ASVs, and a collision-free RESO-based guidance law is developed. Simulation results are used to show the proposed collisionfree guidance law for tracking a maneuvering target.

The contributions of this paper is twofold. First, an antidisturbance guidance law for target tracking is designed based on the reduced-order extended state observer where target dynamics is not required to be known. Second, a collision-free guidance law for target tracking is developed such that the collision between the target and follower vehicle can be avoided. The main features of the proposed guidance law are presented in this paper as follows. First, compared with the target tracking controllers proposed in $[2,27-$ 29] where the velocity of the target should be known in advance, the velocity of the target is not required to be known and only the relative line-of-sight distance and angle between the target and the follower are needed. Second, compared with the target tracking controllers proposed in $[2,10,27-30,34]$ where the collision avoidance problem is not considered, a collision-free RESO-based guidance law is proposed for target tracking of underactuated ASVs where the collision between the target and follower can be avoided.

This paper is organized as follows: Section 2 states the preliminaries and problem formulation. Section 3 gives the target tracking guidance law design and analysis. Section 4 introduces the collision-free target tracking guidance law design and analysis. Section 5 presents the simulation results. Section 6 concludes this paper.

\section{Preliminaries and Problem Formulation}

2.1. Collision Avoidance. In order to assure collision-free target tracking, the following collision avoidance potential functions are introduced [35]:

$$
V_{a}(\rho)=\left(\min \left\{0, \frac{l^{2}-R^{2}}{l^{2}-r^{2}}\right\}\right)^{2},
$$

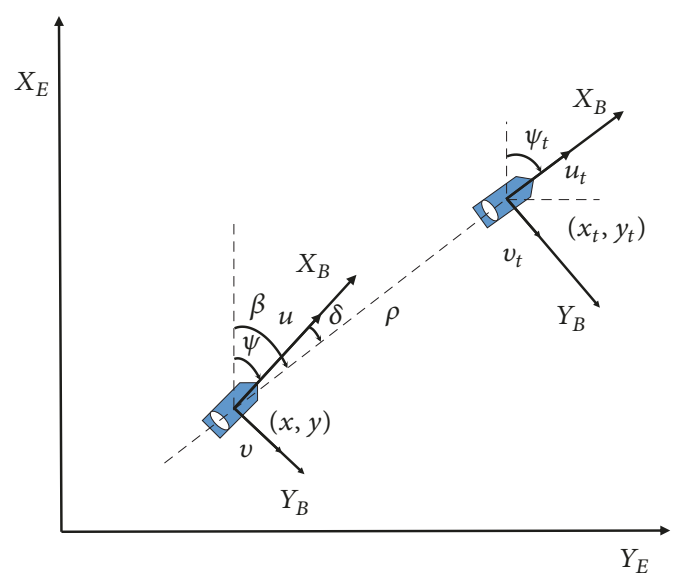

FIGURE 1: A geometrical illustration for tracking a target by an ASV.

where $R>r>0, R$ is the detection region, $r$ is the radius of the avoidance, and $l$ is the distance between the target and follower vehicles defined as

$$
l=\sqrt{\left(y_{t}-y\right)^{2}+\left(x_{t}-x\right)^{2}},
$$

and $x_{t}, y_{t}$ are positions of a target and $x, y$ are positions of a follower.

Function (1) will be infinity when the distance between the vehicle and obstacle approaches avoidance region and is zero outside the detection region. In other words, the function $V_{a}$ will affect the surge velocity when $l$ is inside the detection region.

Taking the partial derivative of the potential function $V_{a}$ with respect to $x$, we can obtain [36]

$$
\begin{cases}\frac{\partial V_{a}}{\partial x}=4 \frac{\left(R^{2}-r^{2}\right)\left(l^{2}-R^{2}\right)}{\left(l^{2}-r^{2}\right)^{3}}\left(x-x_{t}\right), & \text { if } r<l<R \\ 0, & \text { otherwise }\end{cases}
$$

and the partial derivative of function $V_{a}$ with respect to $y$ is

$$
\begin{cases}\frac{\partial V_{a}}{\partial y}=4 \frac{\left(R^{2}-r^{2}\right)\left(l^{2}-R^{2}\right)}{\left(l^{2}-r^{2}\right)^{3}}\left(y-y_{t}\right), & \text { if } r<l<R \\ 0, & \text { otherwise. }\end{cases}
$$

2.2. Vehicle Kinematics. The kinematics of an ASV can be expressed by using an earth-fixed frame $\{E\}$ and a body-fixed frame $\{B\}$ as shown in Figure 1. Let $\left(x_{t}, y_{t}, \psi_{t}\right)$ and $(x, y, \psi)$ be the position and orientation of the target and follower, respectively. $u_{t}, v_{t}, r_{t}$ denote the surge velocity, sway velocity, and angular rate of the target vehicle; $u, v, r$ represent the surge velocity, sway velocity, and angular rate of the follower vehicle, respectively. The kinematics of the target ASV is

$$
\begin{aligned}
& \dot{x}_{t}=u_{t} \cos \psi_{t}-v_{t} \sin \psi_{t}, \\
& \dot{y}_{t}=u_{t} \sin \psi_{t}+v_{t} \cos \psi_{t}, \\
& \dot{\psi}_{t}=r_{t},
\end{aligned}
$$


and the kinematics of follower ASV is

$$
\begin{aligned}
& \dot{x}=u \cos \psi-v \sin \psi, \\
& \dot{y}=u \sin \psi+v \cos \psi, \\
& \dot{\psi}=r .
\end{aligned}
$$

From Figure 1, the line-of-sight range and angle between the target and the follower are defined as

$$
\begin{aligned}
& \rho=\sqrt{\left(y_{t}-y\right)^{2}+\left(x_{t}-x\right)^{2}}, \\
& \beta=\tan ^{-1}\left(\frac{y_{t}-y}{x_{t}-x}\right) .
\end{aligned}
$$

In the following sections, we first consider the target tracking guidance law design being lack of velocity information of the target. Next, the result is extended to collision-free RESO-based guidance law design.

\section{Target Tracking}

At first, the relative dynamics between the target and follower is derived. Then, a RESO is used to estimate the unknown relative dynamics due to the unavailable velocity of the target. Next, an antidisturbance guidance law is designed based on the RESO. Finally, the stability of closed-loop guidance system is analyzed via cascade analysis.

3.1. Relative Dynamics. Two target tracking errors are defined as follows:

$$
\begin{aligned}
& e_{\rho}=\rho-\rho_{d}, \\
& e_{\beta}=\beta-\psi-\delta,
\end{aligned}
$$

where $\rho_{d}$ is a desired range and $\delta=\operatorname{atan} 2(v, u)$ is a sideslip angle. Taking the time derivative of $e_{\rho}$ and $e_{\beta}$ in (8) and using (5) and (6), we have

$$
\begin{aligned}
\dot{e}_{\rho}= & u_{t} \cos \left(\beta-\psi_{t}\right)+v_{t} \sin \left(\beta-\psi_{t}\right)-v \sin (\beta-\psi) \\
& -u \cos (\beta-\psi)-\dot{\rho}_{d}, \\
\dot{e}_{\beta}= & \frac{u_{t} \sin \left(\psi_{t}-\beta\right)+v_{t} \cos \left(\psi_{t}-\beta\right)}{\rho} \\
& -\frac{v \cos (\beta-\psi)+u \sin (\beta-\psi)}{\rho}-\dot{\delta}-r .
\end{aligned}
$$

The control objective of target tracking of ASVs in the presence of unknown target kinematics is to design a surge velocity $u$ and yaw rate $r$ such that

$$
\begin{aligned}
& \lim _{t \longrightarrow \infty}\left|e_{\rho}\right| \leq \epsilon_{1}, \\
& \lim _{t \rightarrow \infty}\left|e_{\beta}\right| \leq \epsilon_{2},
\end{aligned}
$$

for some small constants $\epsilon_{1}$ and $\epsilon_{2}$.
3.2. RESO Design. We first use a RESO to estimate the unknown relative dynamics due to the unavailable velocity of the target. To facilitate controller design, the relative dynamics in (9) is rewritten in the following form:

$$
\begin{aligned}
& \dot{e}_{\rho}=f(\cdot)-u, \\
& \dot{e}_{\beta}=g(\cdot)-r,
\end{aligned}
$$

where

$$
\begin{aligned}
f(\cdot)= & u_{t} \cos \left(\beta-\psi_{t}\right)+v_{t} \sin \left(\beta-\psi_{t}\right) \\
& -v \sin (\beta-\psi)+2 u \sin ^{2}\left(\frac{\beta-\psi}{2}\right)-\dot{\rho}_{d}, \\
g(\cdot)= & \frac{u_{t} \sin \left(\psi_{t}-\beta\right)+v_{t} \cos \left(\psi_{t}-\beta\right)}{\rho} \\
& -\frac{v \cos (\beta-\psi)+u \sin (\beta-\psi)}{\rho}-\dot{\delta} .
\end{aligned}
$$

Since $u_{t}, v_{t}$, and $\psi_{t}$ of the target are not available, $f(\cdot)$ and $g(\cdot)$ are unknown. To address it, a reduced-order extended state observer is proposed as follows [43]:

$$
\begin{aligned}
\dot{p}_{1} & =-k_{1} p_{1}-k_{1}^{2} e_{\rho}+k_{1} u, \\
\hat{f} & =p_{1}+k_{1} e_{\rho}, \\
\dot{p}_{2} & =-k_{2} p_{2}-k_{2}^{2} e_{\beta}+k_{2} r, \\
\hat{g} & =p_{2}+k_{2} e_{\beta},
\end{aligned}
$$

where $p_{1}, p_{2}$ are the auxiliary states of the observer; $k_{1}, k_{2}$ are the observer gains; $\widehat{f}$ and $\hat{g}$ denote the estimation of $f$ and $g$. The initial values of $p_{1}$ and $p_{2}$ are set to $p_{1}\left(t_{0}\right)=-k_{1} e_{\rho}\left(t_{0}\right)$ and $p_{2}\left(t_{0}\right)=-k_{2} e_{\beta}\left(t_{0}\right)$.

Assumption 1. The time derivatives of $f$ and $g$ are bounded by $|\dot{f}| \leq f^{*}$ and $|\dot{g}| \leq g^{*}$, where $f^{*}$ and $g^{*}$ are positive constants.

Since the velocities and accelerations of the target and follower ASVs are naturally bounded, Assumption 1 is reasonable.

The estimation errors are defined as follows:

$$
\begin{aligned}
& \tilde{f}=\widehat{f}-f, \\
& \widetilde{g}=\widehat{g}-g .
\end{aligned}
$$

Taking the derivative of (14) along (13), we have

$$
\begin{aligned}
\dot{\tilde{f}} & =-k_{1} p_{1}-k_{1}^{2} e_{\rho}+k_{1} u+k_{1}(f-u)-\dot{f} \\
& =-k_{1} \tilde{f}-\dot{f} \\
\dot{\tilde{g}} & =-k_{2} p_{2}-k_{2}^{2} e_{\beta}+k_{2} r+k_{3}(g-r)-\dot{g}=-k_{2} \tilde{g}-\dot{g} .
\end{aligned}
$$

The stability of subsystem (15) is stated as follows. 
Lemma 2. Subsystem (15), viewed as a system with the states being $\tilde{f}$ and $\widetilde{g}$ and the inputs being $\dot{f}$ and $\dot{g}$, is input-to-state stability (ISS).

Proof. Construct the following Lyapunov function:

$$
V_{1}=\frac{1}{2} \widetilde{f}^{2}+\frac{1}{2} \widetilde{g}^{2}
$$

Taking the time derivative of $V_{1}$ along (15) results in

$$
\begin{aligned}
\dot{V}_{1} & =-k_{1} \widetilde{f}^{2}-\tilde{f} \dot{f}-k_{2} \widetilde{g}^{2}-\tilde{g} \dot{g} \\
& \leq-\lambda_{\text {min }}\left(K_{1}\right)\left\|E_{1}\right\|^{2}+\|h\|\left\|E_{1}\right\|,
\end{aligned}
$$

where $E_{1}=[\tilde{f}, \tilde{g}]^{T}, K_{1}=\operatorname{diag}\left\{k_{1}, k_{2}\right\}$, and $h=[|\dot{f}|,|\dot{g}|]^{T}$. Noting that

$$
\left\|E_{1}\right\| \geq \frac{|\dot{f}|+|\dot{g}|}{\theta_{1} \lambda_{\min }\left(K_{1}\right)} \geq \frac{\|h\|}{\theta_{1} \lambda_{\min }\left(K_{1}\right)}
$$

renders

$$
\dot{V}_{1} \leq-\lambda_{\text {min }}\left(K_{1}\right)\left(1-\theta_{1}\right)\left\|E_{1}\right\|^{2},
$$

where $0<\theta_{1}<1$. As a consequence, subsystem (15) is ISS, and

$$
\begin{aligned}
& \left\|E_{1}(t)\right\| \\
& \quad \leq \max \left\{\sigma_{o}\left(\left\|E_{1}(0)\right\|, t\right), \gamma_{o 1}(|\dot{f}|)+\gamma_{o 2}(|\dot{g}|)\right\},
\end{aligned}
$$

where $\sigma_{o}$ is a class $\mathscr{K} \mathscr{L}$ function, $\gamma_{o 1}, \gamma_{o 2}$ are the class $\mathscr{K}$ function, and

$$
\gamma_{o 1}(s)=\gamma_{o 2}(s)=\frac{s}{\theta_{1} \lambda_{\min }\left(K_{1}\right)} .
$$

3.3. Guidance Law Design. Based on the estimated terms $\widehat{f}$ and $\widehat{g}$ from the RESO, an antidisturbance guidance law is proposed as follows:

$$
\begin{aligned}
& u=\frac{k_{3} e_{\rho}}{\sqrt{\left\|e_{\rho}\right\|^{2}+\Delta_{1}^{2}}}+\hat{f}, \\
& r=\frac{k_{4} e_{\beta}}{\sqrt{\left\|e_{\beta}\right\|^{2}+\Delta_{2}^{2}}}+\hat{g},
\end{aligned}
$$

where $k_{3}$ and $k_{4}$ are positive constants; $\Delta_{1}$ and $\Delta_{2}$ are positive constants, which are used to limit the maximum value of control laws.

Substituting (14) and (22) into (11) results in

$$
\begin{gathered}
\dot{e}_{\rho}=-\frac{k_{3} e_{\rho}}{\sqrt{\left\|e_{\rho}\right\|^{2}+\Delta_{1}^{2}}}-\tilde{f}, \\
\dot{e}_{\beta}=-\frac{k_{4} e_{\beta}}{\sqrt{\left\|e_{\beta}\right\|^{2}+\Delta_{2}^{2}}}-\tilde{g} .
\end{gathered}
$$

The ISS property of subsystem (23) is stated as follows.
Lemma 3. Subsystem (23), viewed as a system with the states being $e_{\rho}$ and $e_{\beta}$ and the inputs being $\tilde{f}$ and $\tilde{g}$, is ISS.

Proof. Construct the following Lyapunov function:

$$
V_{2}=\frac{1}{2} e_{\rho}^{2}+\frac{1}{2} e_{\beta}^{2} .
$$

Taking the time derivative of $V_{2}$ along (23) results in

$$
\begin{aligned}
\dot{V}_{2} & =-\frac{k_{3} e_{\rho}^{2}}{\sqrt{\left\|e_{\rho}\right\|^{2}+\Delta_{1}^{2}}}-\tilde{f} e_{\rho}-\frac{k_{4} e_{\beta}^{2}}{\sqrt{\left\|e_{\beta}\right\|^{2}+\Delta_{2}^{2}}}-\tilde{g} e_{\beta} \\
& \leq-\frac{\lambda_{\min }\left(K_{2}\right)\left\|E_{2}\right\|^{2}}{\sqrt{\left\|E_{2}\right\|^{2}+\Delta_{\max _{1}}^{2}}}+\|w\|\left\|E_{2}\right\|,
\end{aligned}
$$

where $E_{2}=\left[e_{\rho}, e_{\beta}\right]^{T}, K_{2}=\operatorname{diag}\left\{k_{3}, k_{4}\right\}, w=[|\tilde{f}|,|\widetilde{g}|]^{T}$, and $\Delta_{\max _{1}}=\max \left\{\Delta_{1}, \Delta_{2}\right\}$.

Noting that

$$
\frac{\left\|E_{2}\right\|}{\sqrt{\left\|E_{2}\right\|+\Delta_{\max }^{2}}} \geq \frac{|\tilde{f}|+|\tilde{g}|}{\theta_{2} \lambda_{\min }\left(K_{2}\right)} \geq \frac{\|w\|}{\theta_{2} \lambda_{\min }\left(K_{2}\right)}
$$

renders

$$
\dot{V}_{2} \leq-\frac{\lambda_{\min }\left(K_{2}\right)\left(1-\theta_{2}\right)\left\|E_{2}\right\|^{2}}{\sqrt{\left\|E_{2}\right\|^{2}+\Delta_{\max _{1}}^{2}}},
$$

where $0<\theta_{2}<1$. As a consequence, subsystem (23) is ISS, and

$$
\begin{aligned}
& \left\|E_{2}(t)\right\| \\
& \quad \leq \max \left\{\sigma_{c}\left(\left\|E_{2}(0)\right\|, t\right), \gamma_{c 1}(|\widetilde{f}|)+\gamma_{c 2}(|\widetilde{g}|)\right\},
\end{aligned}
$$

where $\sigma_{c}$ is a class $\mathscr{K} \mathscr{L}$ function, $\gamma_{c 1}$ and $\gamma_{c 2}$ are the class $\mathscr{K}$ functions, and

$$
\gamma_{c 1}(s)=\gamma_{c 2}(s)=\mu^{-1}\left(\frac{s}{\theta_{2} \lambda_{\min }\left(K_{2}\right)}\right),
$$

with $\mu=|s| / \sqrt{s^{2}+\Delta_{\text {max }_{1}}^{2}}$.

The proposed guidance law can be augmented with other methods such as PID control, adaptive control [44, 45], sliding mode control [46], and robust control at the kinetic level for achieving the desired target tracking control performance.

3.4. Stability Analysis. To analyze the stability of the entire closed-loop guidance system, rewrite the disturbance estimation subsystem (15) and target tracking error subsystem (23) in a compact form as

$$
\Sigma_{1}:\left\{\begin{array}{l}
\dot{e}_{\rho}=-\frac{k_{3} e_{\rho}}{\sqrt{\left\|e_{\rho}\right\|^{2}+\Delta_{1}^{2}}}-\tilde{f}, \\
\dot{e}_{\beta}=\frac{k_{4} e_{\beta}}{\sqrt{\left\|e_{\beta}\right\|^{2}+\Delta_{2}^{2}}}-\tilde{g},
\end{array}\right.
$$


and

$$
\Sigma_{2}:\left\{\begin{array}{l}
\dot{\tilde{f}}=-k_{1} \tilde{f}-\dot{f} \\
\dot{\tilde{g}}=-k_{2} \widetilde{g}-\dot{g}
\end{array}\right.
$$

The stability of the cascade of subsystem $\Sigma_{1}$ and subsystem $\Sigma_{2}$ is given by the following theorem.

Theorem 4. Under Assumption 1, the closed-loop system cascaded by subsystem (15) and subsystem (23) is ISS, and the target tracking errors converge to a small neighborhood of the origin.

Proof. Subsystem $\Sigma_{1}$ with states $\left(e_{\rho}, e_{\beta}\right)$ and exogenous inputs $(\tilde{f}, \tilde{g})$ and subsystem $\Sigma_{2}$ with states $(\tilde{f}, \tilde{g})$ and exogenous inputs $\dot{f}$ and $\dot{g}$ are ISS. By Lemma C.4 in [47], it follows that the cascade systems $\Sigma_{1}$ and $\Sigma_{2}$ with states $\left(e_{\rho}, e_{\beta}, \tilde{f}, \tilde{g}\right)$ and exogenous inputs $(\dot{f}, \dot{g})$ are ISS, i.e., there exist class $\mathscr{K} \mathscr{L}$ function $\sigma_{1}$ and $\mathscr{K}$ function $\gamma_{c 1}, \gamma_{o 1}, \gamma_{c 2}$, and $\gamma_{o 2}$, such that

$$
\begin{aligned}
& \left\|E_{a}\right\| \leq \max \left\{\sigma_{1}\left(\left\|E_{a}(0)\right\|, t\right), \gamma_{c 1} \circ \gamma_{o 1}(|\dot{f}|)+\gamma_{c 2}\right. \\
& \quad \circ \gamma_{o 2}(|\dot{g}|\},
\end{aligned}
$$

where $E_{a}=\left[e_{\rho}, e_{\beta}, \tilde{f}, \tilde{g}\right]$. As $t \longrightarrow \infty, \sigma_{1} \longrightarrow \infty$, it follows that

$$
\begin{aligned}
\lim _{t \rightarrow \infty}\left\|E_{a}\right\| \leq & \gamma_{c 1} \circ \gamma_{o 1}(|\dot{f}|)+\gamma_{c 2} \circ \gamma_{o 2}(|\dot{g}|) \\
\leq & \mu^{-1}\left(\frac{f^{*}}{\theta_{1} \theta_{2} \lambda_{\min }\left(K_{1}\right) \lambda_{\min }\left(K_{2}\right)}\right) \\
& +\mu^{-1}\left(\frac{g^{*}}{\theta_{1} \theta_{2} \lambda_{\min }\left(K_{1}\right) \lambda_{\min }\left(K_{2}\right)}\right),
\end{aligned}
$$

implying (10) with $\mu=|s| / \sqrt{s^{2}+\Delta_{\max _{1}}^{2}}$. Note that $|\dot{g}|$ and $|\dot{f}|$ are bounded by $g^{*}$ and $f^{*}$. Then, the errors $e_{\rho}, e_{\beta}, \tilde{f}$ and $\tilde{g}$ are all bounded. Note that only uniform ultimate boundedness of closed-loop system can be achieved due to the existence of $\dot{g}$ and $\dot{f}$. If $\dot{g}=0$ and $\dot{f}=0$, the closed-loop system is asymptotical stable.

\section{Collision-Free Target Tracking}

4.1. Guidance Law Design. In previous section, a target tracking controller is developed for ASVs without using the velocity of the target. In this section, a collision-free target tracking controller is developed based on a RESO and an artificial potential function. To achieve a collision-free target tracking, a desired orientation is defined as follows:

$$
\theta_{r}=\operatorname{atan} 2\left(y_{t}-y-\frac{\partial V_{a}}{\partial y}, x_{t}-x-\frac{\partial V_{a}}{\partial x}\right),
$$

and the angle tracking error is redefined as

$$
e_{\beta}=\theta_{r}-\psi-\delta
$$

The guidance law for collision-free target tracking based on RESO is designed as follows:

$$
\begin{aligned}
& u=\frac{k_{5}\left(e_{\rho}+\partial V_{a} / \partial e_{\rho}\right)}{\sqrt{\left(e_{\rho}+\partial V_{a} / \partial e_{\rho}\right)^{2}+\Delta_{3}^{2}}}+\widehat{f}, \\
& r=\frac{k_{6} e_{\beta}}{\sqrt{e_{\beta}^{2}+\Delta_{4}^{2}}}+\widehat{g},
\end{aligned}
$$

where $k_{5}$ and $k_{6}$ are positive constants; $\Delta_{3}$ and $\Delta_{4}$ are positive constants, which are employed to limit the maximum value of control laws.

Substituting (14) and (36) into (11) results in

$$
\begin{aligned}
& \dot{e}_{\rho}=-\frac{k_{5}\left(e_{\rho}+\partial V_{a} / \partial e_{\rho}\right)}{\sqrt{\left(e_{\rho}+\partial V_{a} / \partial e_{\rho}\right)^{2}+\Delta_{3}^{2}}}-\tilde{f}, \\
& \dot{e}_{\beta}=-\frac{k_{6} e_{\beta}}{\sqrt{e_{\beta}^{2}+\Delta_{4}^{2}}}-\tilde{g} .
\end{aligned}
$$

The ISS property of subsystem (37) is stated as follows.

Lemma 5. Subsystem (37), viewed as a system with the states being $e_{\rho}+\partial V_{a} / \partial e_{\rho}$ and $e_{\beta}$ and the inputs being $\tilde{f}$ and $\tilde{g}$, is ISS.

Proof. Construct a Lyapunov function for system (37) as

$$
V_{3}=\frac{1}{2} e_{\rho}^{2}+\frac{1}{2} e_{\beta}^{2}+V_{a}
$$

Taking the time derivative of $V_{3}$ along (37), (3), and (4), we have

$$
\begin{aligned}
\dot{V}_{3}= & -\frac{k_{5}\left(e_{\rho}+\partial V_{a} / \partial e_{\rho}\right)^{2}}{\sqrt{\left(e_{\rho}+\partial V_{a} / \partial e_{\rho}\right)^{2}+\Delta_{3}^{2}}}+\tilde{f}\left(e_{\rho}+\frac{\partial V_{a}}{\partial e_{\rho}}\right) \\
& -\frac{k_{6} e_{\beta}^{2}}{\sqrt{e_{\beta}^{2}+\Delta_{4}^{2}}}+\widetilde{g} e_{\beta} \\
\leq & -\frac{\lambda_{\min _{1}}\left(K_{3}\right)\left\|E_{3}\right\|^{2}}{\sqrt{\left\|E_{3}\right\|^{2}+\Delta_{\max _{2}}^{2}}+\|w\|\left\|E_{3}\right\|,}
\end{aligned}
$$

where $E_{3}=\left[e_{\rho}+\partial V_{a} / \partial e_{\rho}, e_{\beta}\right]^{T}, K_{3}=\operatorname{diag}\left\{k_{5}, k_{6}\right\}$, and $\Delta_{\max _{2}}=\max \left\{\Delta_{3}, \Delta_{4}\right\}$.

Noting that

$$
\frac{\left\|E_{3}\right\|}{\sqrt{\left\|E_{3}\right\|^{2}+\Delta_{\max _{2}}^{2}}} \geq \frac{|\tilde{f}|+|\tilde{g}|}{\theta_{3} \lambda_{\min }\left(K_{3}\right)} \geq \frac{\|w\|}{\theta_{3} \lambda_{\min }\left(K_{3}\right)}
$$

renders

$$
\dot{V}_{3} \leq-\frac{\lambda_{\min }\left(K_{3}\right)\left(1-\theta_{3}\right)\left\|E_{3}\right\|^{2}}{\sqrt{\left\|E_{3}\right\|^{2}+\Delta_{\max _{2}}^{2}}},
$$


where $0<\theta_{3}<1$. As a consequence, subsystem (37) is ISS. Since $\dot{V}_{3}$ is negative definite, then $V_{3}$ is not increasing inside the detection region. Since

$$
\lim _{\left\|z-z_{a}\right\| \rightarrow r^{+}} V_{a}=\infty,
$$

where $z=[x, y]^{T}$ and $z_{a}=\left[x_{t}, y_{t}\right]^{T}$, then collision avoidance is guaranteed.

4.2. Stability Analysis. Finally, we analyze the cascade stability of subsystem (15) and subsystem (37) in a compact form:

$$
\Sigma_{3}:\left\{\begin{array}{l}
\dot{e}_{\rho}=-\frac{k_{5}\left(e_{\rho}+\partial V_{a} / \partial e_{\rho}\right)}{\sqrt{\left(e_{\rho}+\partial V_{a} / \partial e_{\rho}\right)^{2}+\Delta_{3}^{2}}}-\tilde{f} \\
\dot{e}_{\beta}=-\frac{k_{8} e_{\beta}}{\sqrt{e_{\beta}^{2}+\Delta_{4}^{2}}}-\tilde{g},
\end{array}\right.
$$

and

$$
\Sigma_{4}:\left\{\begin{array}{l}
\dot{\tilde{f}}=-k_{1} \tilde{f}-\dot{f}, \\
\dot{\tilde{g}}=-k_{2} \tilde{g}-\dot{g} .
\end{array}\right.
$$

The following theorem presents the stability of the cascade system consisting of subsystem (15) and subsystem (37).

Theorem 6. Consider the closed-loop guidance system consisting of follower ASV with kinematics (6), the target ASV with kinematics (5), and the guidance law (36) (13). If Assumption 1 is satisfied, the proposed guidance law can achieve the control objective described in Section 3.1. The closed-loop system cascaded by subsystem (15) and subsystem (37) is ISS. Besides,

(1) outside the detection range, the tracking errors $\left(e_{\rho}, e_{\beta}\right)$ converge to a small neighborhood of the origin,

(2) inside the detection range, collision avoidance is guaranteed, i.e., for all $t \geq 0,\left\|[x, y]^{T}-\left[x_{t}, y_{t}\right]^{T}\right\|-r \geq \epsilon_{4}$ for some constant $\epsilon_{4}$.

Proof. Part A. The cascade system consisting of subsystem $\Sigma_{3}$ and subsystem $\Sigma_{4}$ with the relative distance $\rho(\rho>R)$ is ISS. The proof is the same as the proof of Theorem 4 .

Part $B$. The cascade system consisting of subsystem $\Sigma_{3}$ and subsystem $\Sigma_{4}$ with the relative distance $\rho(\rho<r)$ is ISS. It has been proved that subsystem $\Sigma_{3}$ with states $\left(e_{\rho}+\partial V_{a} / \partial e_{\rho}, e_{\beta}\right)$ and exogenous inputs $(\tilde{f}, \widetilde{g})$ and subsystem $\Sigma_{4}$ with states $(\tilde{f}$, $\widetilde{g})$ and exogenous inputs $\dot{f}$ and $\dot{g}$ are ISS. By Lemma C.4 [47], it follows that the cascade systems $\Sigma_{3}$ and $\Sigma_{4}$ with states $\left(e_{\rho}+\right.$ $\left.\partial V_{a \rho} / \partial e_{\rho}, e_{\beta}, \tilde{f}, \widetilde{g}\right)$ and exogenous inputs $(\dot{f}, \dot{g})$ are ISS. Note that the errors $e_{\rho}+\partial V_{a \rho} / \partial e_{\rho}, e_{\beta}, \tilde{f}$, and $\widetilde{g}$ are all bounded. Then, collision avoidance is guaranteed $\left\|[x, y]^{T}-\left[x_{t}, y_{t}\right]^{T}\right\| \geq$ $r_{d}$ for all $t \geq 0$, where $r_{d}=r+\epsilon_{4}$ with $\epsilon_{4}$ being a constant.

A parameter selection guideline is provided as follows. The parameters $k_{1}$ and $k_{2}$ determine the speed of observer. The parameters $k_{3}, k_{4}, k_{5}$, and $k_{6}$ can be chosen according to the desired response of the closed-loop system. The parameters $\Delta_{1}, \Delta_{2}, \Delta_{3}$, and $\Delta_{4}$ can be used to regulate the transient performance.

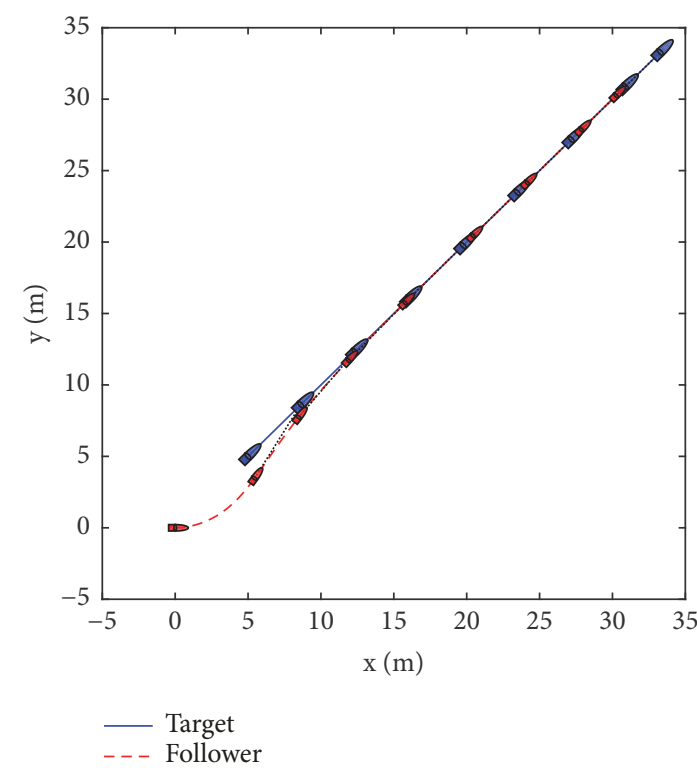

FIGURE 2: Trajectory of target and follower ASV.

\section{Simulation Results}

In this section, simulation results are given to show the performance of the proposed guidance law for collision-free target tracking. Two cases are presented: (1) the velocity of target is constant; (2) the velocity of target is time-varying. The vehicle tracks the target with relative distance $\rho_{d}\left(\rho_{d}>\right.$ $\left.r>R t<40 s, \rho_{d}<r<R t>40 s\right)$. The initial configurations of the follower and target vehicle are $(x, y, \psi)(0)=(0,0,0)$ and $\left(x_{t}, y_{t}, \psi_{t}\right)(0)=(5,5, \pi / 4)$, respectively. The control parameters are chosen as follows: $k_{1}=5, k_{2}=5, k_{3}=0.1$, $k_{4}=0.2, k_{5}=0.1, k_{6}=0.2, \Delta_{1}=0.5, \Delta_{2}=0.5, \Delta_{3}=0.5$, $\Delta_{4}=0.5, r=3 m, R=5 m, \rho_{d}=6 m(t<40 s)$, and $\rho_{d}=2 m(t>40 s)$.

5.1. Target Tracking and Collision Avoidance with a Target of Constant Velocity. Consider the ASV model (6) controlled by the guidance law (22) with constant velocity $u_{t}=0.5 \mathrm{~m} / \mathrm{s}$ and $r_{t}=0 \mathrm{rad} / \mathrm{s}$. Simulation results are shown in Figures $2-5$, and Figure 2 shows the trajectories of target and follower ASV. It reveals that the follower vehicle heads for the target in a short time while holding a desired distance although the target dynamics is uncertain. Figure 3 shows that the follower vehicle tracks the target with distance $\rho_{d}=6 m$ when the distance satisfies $\rho_{d}>r>R$. After $40 \mathrm{~s}$, the distance $\rho_{d}$ is changed from $6 m$ to $2 m$, and the tracking distance becomes $R>\rho_{d}>r$. It reveals that the proposed guidance law can achieve collision avoidance well. The output of RESO and the relative dynamics of target are compared in Figure 4. It shows that the RESO can compensate the uncertain target kinematics efficiently. Figure 5 shows the guidance signals of the proposed guidance law. It reveals that the guidance signals are all bounded.

5.2. Target Tracking and Collision Avoidance with a Target of Time-Varying Velocity. The guidance law (36) is employed 


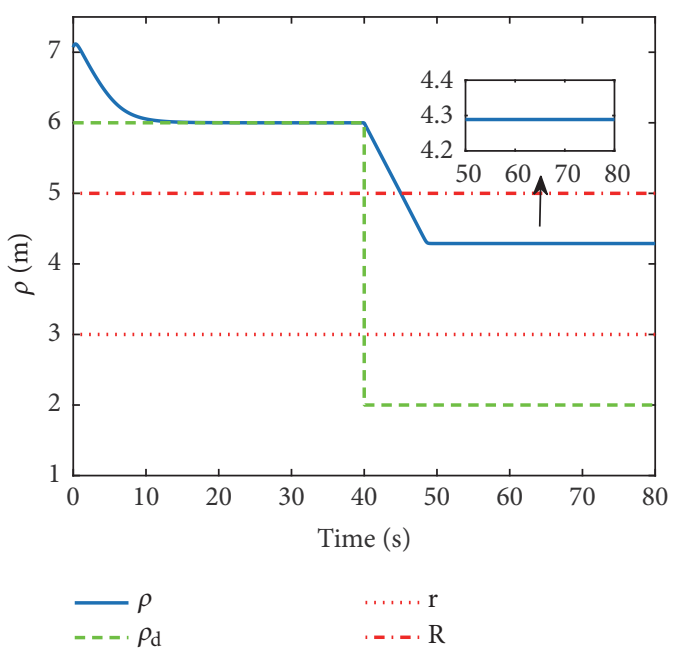

FIGURE 3: The distance between vehicle and target.
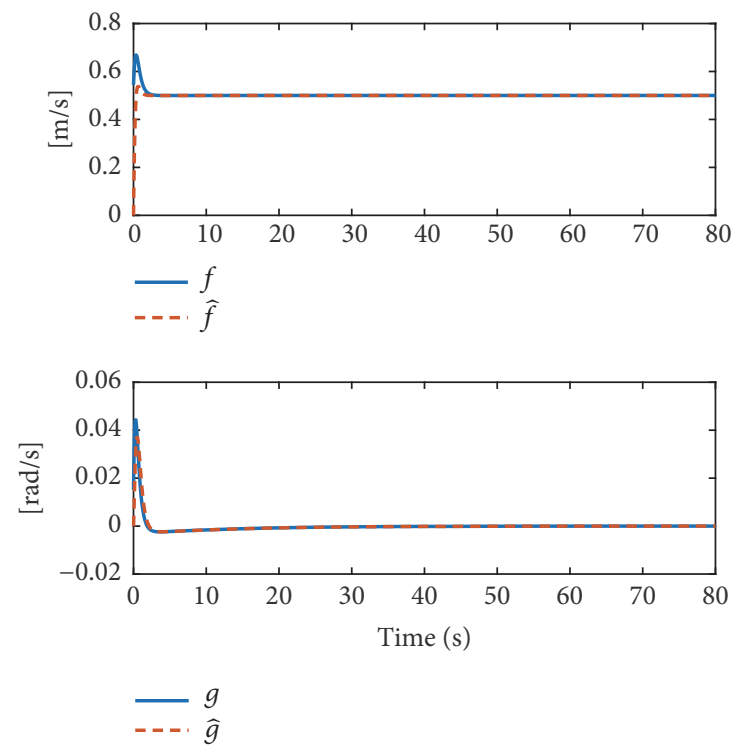

FIGURE 4: Estimation performance of the RESOs.
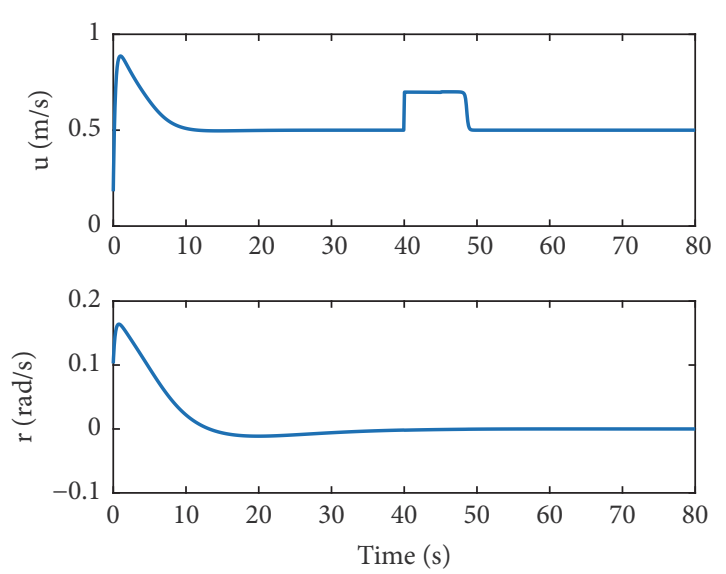

FIGURE 5: Guidance law.

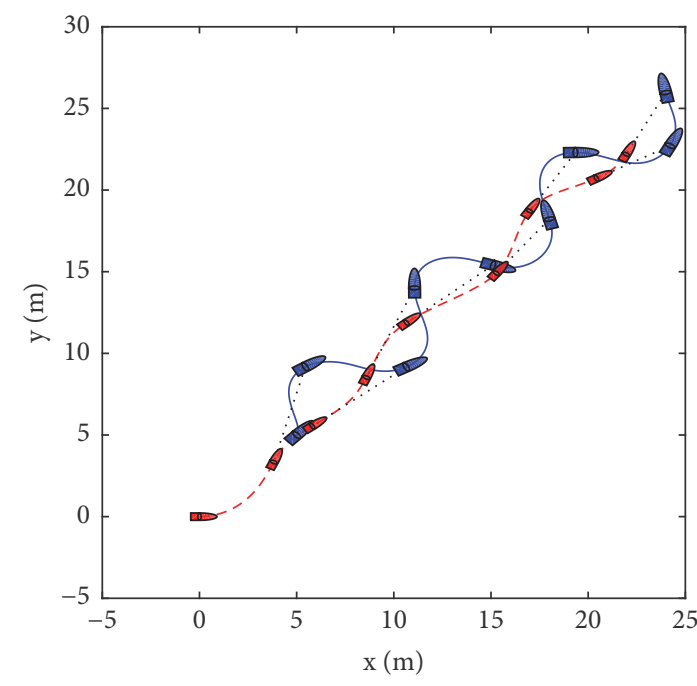

— Target

- - - Follower

FIGURE 6: Trajectory of target and follower ASV.

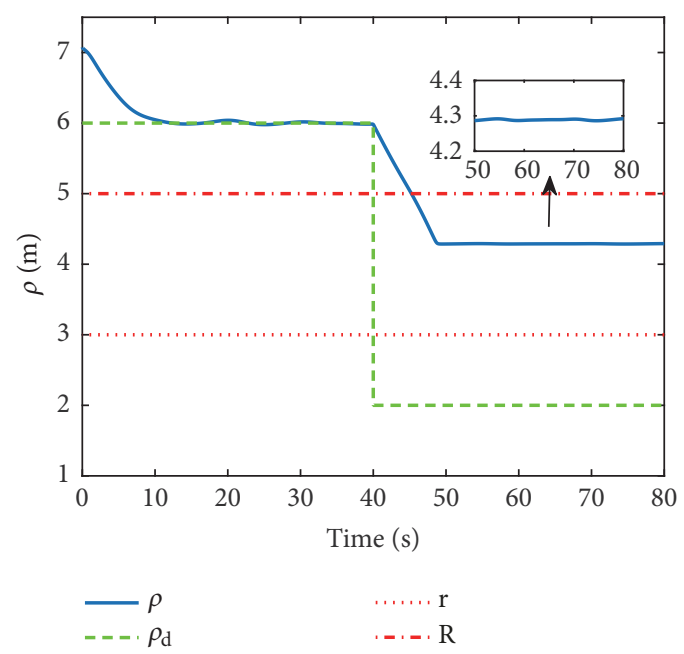

FIgURE 7: Target tracking errors.

to track a target with time-varying velocity $u_{t}=(2+$ $0.1 \sin (2 t)) \mathrm{m} / \mathrm{s}$ and $r_{t}=0.5 \sin (2 t) \mathrm{rad} / \mathrm{s}$. Figures $6-9$ show the simulation results. Figure 6 shows that the follower vehicle can track the target with given relative distance. The relative distance is shown in Figure 7. It implies that the follower vehicle tracks the target with given distance $\rho_{d}$ when the distance is designed as $\rho_{d}>r>R$. After $40 s$, the distance $\rho_{d}$ is changed from $6 m$ to $2 m$. The tracking distance becomes $R>\rho_{d}>r$ rather than the given distance $2 m$. It indicates that the proposed guidance law for target tracking extended to collision avoidance is effective with the time-varying velocity of target. The output of the RESO and relative dynamics of target are compared in Figure 8. It shows that the RESO can compensate the uncertain target dynamics efficiently. Figure 9 shows the guidance signals of the proposed guidance law. It observed that the guidance signals are all bounded. 

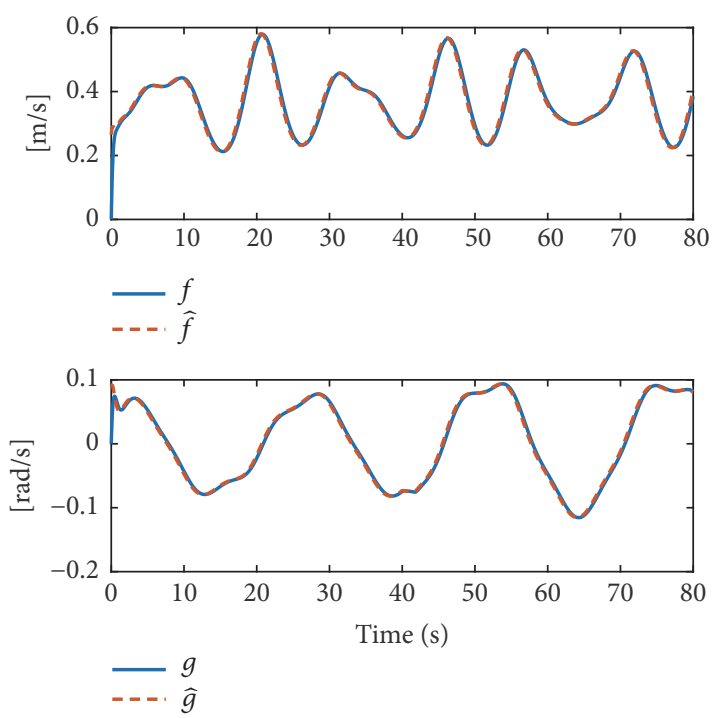

FIGURE 8: Estimation performance of the RESOs.
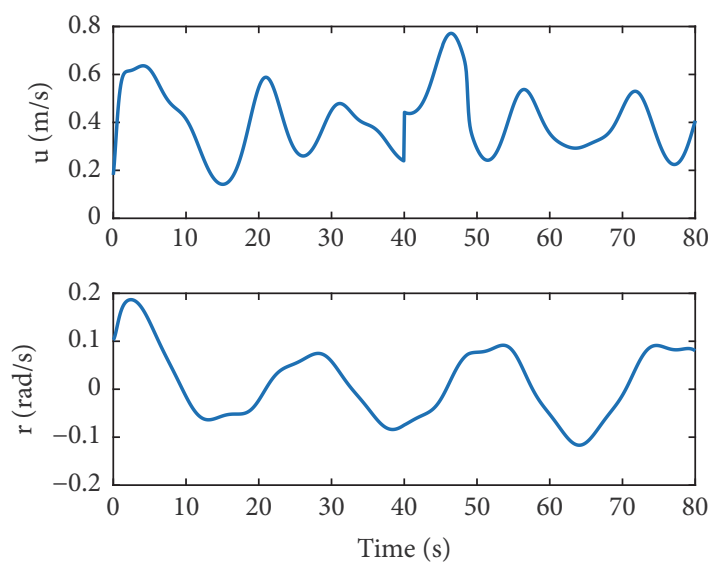

FIGURE 9: Guidance law.

\section{Conclusions}

This paper considers the target tracking problem of an ASV in the presence of a maneuvering target where the velocity information of the target is totally unknown to the follower ASV. A reduced-order extended state observer is used to estimate the unknown relative dynamics. Then, an antidisturbance guidance law is developed based on the reduced-order extended state observer for target tracking. The stability of closed-loop target tracking guidance system is analyzed via cascade analysis. Finally, the result is extended to the case that collisions between the target and leader are avoided during tracking, and a collision-free target tracking guidance law is developed. Simulation results verify the effectiveness of the proposed guidance law for tracking a maneuvering target with arbitrary velocity and having the ability to avoid collision in the presence of a maneuvering target.

\section{Data Availability}

The data used to support the findings of this study are included within the article.

\section{Conflicts of Interest}

The authors declare that they have no conflicts of interest.

\section{Acknowledgments}

Zhouhua Peng and Dan Wang were supported in part by the National Natural Science Foundation of China under Grants 51579023 and 61673081, in part by the Innovative Talents in Universities of Liaoning Province under Grant LR2017014, in part by High Level Talent Innovation and Entrepreneurship Program of Dalian under Grant 2016RQ036, in part by the National Key Research and Development Program of China under Grant 2016YFC0301500, and in part by the Fundamental Research Funds for the Central Universities under Grant 3132016313.

\section{References}

[1] T. I. Fossen, Handbook of Marine Craft Hydrodynamics and Motion Control, John Wiley \& Sons Ltd, 2011.

[2] R. Cui, S. S. Ge, B. Voon Ee How, and Y. Sang Choo, "Leaderfollower formation control of underactuated autonomous underwater vehicles," Ocean Engineering, vol. 37, no. 17-18, pp. 1491-1502, 2010.

[3] X. Xiang, C. Yu, and Q. Zhang, "On intelligent risk analysis and critical decision of underwater robotic vehicle," Ocean Engineering, vol. 140, no. 1, pp. 453-465, 2017.

[4] R. Cui, C. Yang, Y. Li, and S. Sharma, "Adaptive Neural Network Control of AUVs With Control Input Nonlinearities Using Reinforcement Learning," IEEE Transactions on Systems, Man, and Cybernetics: Systems, vol. 47, no. 6, pp. 1019-1029, 2017.

[5] Z. Peng, J. Wang, and J. Wang, "Constrained control of autonomous underwater vehicles based on command optimization and disturbance estimation," IEEE Transactions on Industrial Electronics, 2018.

[6] X. B. Xiang, L. Lapierre, and B. Jouvencel, "Smooth transition of AUV motion control: from fully-actuated to under-actuated configuration," Robotics and Autonomous Systems, vol. 67, no. 5, pp. 14-22, 2015.

[7] Y. Zhang, D. Wang, and Z. Peng, "Consensus maneuvering for a class of nonlinear multivehicle systems in strict-feedback form," IEEE Transactions on Cybernetics, 2018.

[8] L. Liu, D. Wang, and Z. Peng, "Coordinated path following of multiple underacutated marine surface vehicles along one curve," ISA Transactions, vol. 64, pp. 258-268, 2016.

[9] X. Xiang, C. Yu, and Q. Zhang, "Robust fuzzy 3D path following for autonomous underwater vehicle subject to uncertainties," Computers Operations Research, vol. 84, pp. 165-177, Aug 2017.

[10] L. Liu, D. Wang, and Z. Peng, "ESO-Based Line-of-Sight Guidance Law for Path Following of Underactuated Marine Surface Vehicles with Exact Sideslip Compensation," IEEE Journal of Oceanic Engineering, vol. 42, no. 2, pp. 477-487, 2017.

[11] Z. Peng and J. Wang, "Output-feedback path-following control of autonomous underwater vehicles based on an extended state 
observer and projection neural networks," IEEE Transactions on Systems, Man, and Cybernetics: Systems, vol. 48, no. 4, pp. 535544, 2018.

[12] L. Liu, D. Wang, Z. Peng, and H. Wang, "Predictor-based LOS guidance law for path following of underactuated marine surface vehicles with sideslip compensation," Ocean Engineering, vol. 124, pp. 340-348, 2016.

[13] Z. Zheng and M. Feroskhan, "Path following of a surface vessel with prescribed performance in the presence of input saturation and external disturbances," IEEE/ASME Transactions on Mechatronics, vol. 22, no. 6, pp. 2564-2575, 2017.

[14] Z. Zheng and L. Sun, "Error constrained LOS path following of a surface vessel with actuator saturation and faults," IEEE Transactions on Systems, Man, and Cybernetics: Systems, 2018.

[15] Z. Yan, Y. Liu, J. Zhou, and D. Wu, "Path following control of an AUV under the current using the SVR-ADRC", Journal of Applied Mathematics, vol. 2014, Article ID 476419, 12 pages, 2014.

[16] J. Zhou, Z. Tang, H. Zhang, and J. Jiao, "Spatial path following for AUVs using adaptive neural network controllers," Mathematical Problems in Engineering, vol. 2013, Article ID 749689, 9 pages, 2013

[17] Z. Peng, J. Wang, and D. Wang, "Distributed Maneuvering of Autonomous Surface Vehicles Based on Neurodynamic Optimization and Fuzzy Approximation," IEEE Transactions on Control Systems Technology, vol. 26, no. 3, pp. 1083-1090, 2018.

[18] L. Liu, D. Wang, Z. Peng, and T. Li, "Modular adaptive control for LOS-based cooperative path maneuvering of multiple underactuated autonomous surface vehicles," IEEE Transactions on Systems, Man, and Cybernetics: Systems, vol. 47, no. 7, pp. 1613-1624, 2017.

[19] Z. Peng, J. Wang, and D. Wang, "Containment maneuvering of marine surface vehicles with multiple parameterized paths via spatial-temporal decoupling," IEEE/ASME Transactions on Mechatronics, vol. 22, no. 2, pp. 1026-1036, 2017.

[20] Z. Peng, J. Wang, and D. Wang, "Distributed containment maneuvering of multiple marine vessels via neurodynamicsbased output feedback," IEEE Transactions on Industrial Electronics, vol. 64, no. 5, pp. 3831-3839, 2017.

[21] Y. Wang and Y. Yao, "Consensus Path-following Control of Multiple Underactuated Unmanned Underwater Vehicles," Complexity, 2018.

[22] X. Xiang, C. Yu, Z. Niu, and Q. Zhang, "Subsea cable tracking by autonomous underwater vehicle with magnetic sensing guidance," Sensors, vol. 16, no. 8, 2016.

[23] M. A. Santos, B. S. Rego, G. V. Raffo, and A. Ferramosca, "Suspended Load Path Tracking Control Strategy Using a Tilt-Rotor UAV," Journal of Advanced Transportation, vol. 2017, Article ID 9095324, 22 pages, 2017.

[24] B. Braginsky and H. Guterman, "Trajectory controller for Autonomous Surface Vehicle under sea waves," Oceans, pp. 1$5,2015$.

[25] Z. Yin, W. Â He, C. Â Yang, and S. Changyin, "Control design of a marine vessel system using reinforcement learning," Neurocomputing, vol. 311, pp. 353-362, Oct 2018.

[26] Z. Zheng, Y. Huang, L. Xie, and B. Zhu, "Adaptive Trajectory Tracking Control of a Fully Actuated Surface Vessel With Asymmetrically Constrained Input and Output," IEEE Transactions on Control Systems Technology, vol. 26, no. 5, pp. 1851-1859, 2018.
[27] M. Breivik, V. E. Hovstein, and T. I. Fossen, "Straight-line target tracking for unmanned surface vehicles," Modeling, Identification and Control: A Norwegian Research Bulletin, vol. 29, no. 4, pp. 131-149, 2008.

[28] K. Shojaei, "Leader-follower formation control of underactuated autonomous marine surface vehicles with limited torque," Ocean Engineering, vol. 105, pp. 196-205, 2015.

[29] X. Jin, "Fault tolerant finite-time leader-follower formation control for autonomous surface vessels with LOS range and angle constraints," Automatica, vol. 68, pp. 228-236, 2016.

[30] F. Fahimi, "Sliding-mode formation control for underactuated surface vessels," IEEE Transactions on Robotics, vol. 23, no. 3, pp. 617-622, 2007.

[31] Z. Peng, D. Wang, Z. Chen, X. Hu, and W. Lan, "Adaptive dynamic surface control for formations of autonomous surface vehicles with uncertain dynamics," IEEE Transactions on Control Systems Technology, vol. 21, no. 2, pp. 513-520, 2013.

[32] S.-L. Dai, M. Wang, and C. Wang, "Neural learning control of marine surface vessels with guaranteed transient tracking performance," IEEE Transactions on Industrial Electronics, vol. 63, no. 3, pp. 1717-1727, 2016.

[33] O. Namaki-Shoushtari, A. P. Aguiar, and A. Khaki-Sedigh, "Target tracking of autonomous robotic vehicles using rangeonly measurements: a switched logic-based control strategy," International Journal of Robust and Nonlinear Control, vol. 22, no. 17, pp. 1983-1998, 2012.

[34] L. Liu, D. Wang, Z. Peng, and C. L. P. Chen, "Bounded neural network control for target tracking of underactuated autonomous surface vehicles in the presence of uncertain target dynamics," IEEE Transactions on Neural Networks and Learning Systems, 2018.

[35] S. Mastellone, D. M. Stipanović, C. R. Graunke, K. A. Intlekofer, and M. W. Spong, "Formation control and collision avoidance for multi-agent non-holonomic systems: Theory and experiments," International Journal of Robotics Research, vol. 27, no. 1, pp. 107-126, 2008.

[36] S. J. Yoo, "Adaptive neural tracking and obstacle avoidance of uncertain mobile robots with unknown skidding and slipping," Information Sciences, vol. 238, pp. 176-189, 2013.

[37] J. Han, "Extended state observer for a class of uncertain plants," Control and Decision, vol. 10, no. 1, pp. 85-88, 1995 (Chinese).

[38] J. Han, Active Disturbance Rejection Control Technique The Technique for Estimating and Compensating the Uncertainties, National Defense Industry Press, Beijing, China, 2008.

[39] J. Q. Han, "From PID to active disturbance rejection control," IEEE Transactions on Industrial Electronics, vol. 56, no. 3, pp. 900-906, 2009.

[40] B.-Z. Guo and Z. liang Zhao, "On the convergence of an extended state observer for nonlinear systems with uncertainty," Systems Control Letters, vol. 60, no. 6, pp. 420-430, Jun 2011.

[41] R. Cui, X. Zhang, and D. Cui, "Adaptive sliding-mode attitude control for autonomous underwater vehicles with input nonlinearities," Ocean Engineering, vol. 123, pp. 45-54, 2016.

[42] R. Cui, L. Chen, C. Yang, and M. Chen, "Extended State Observer-Based Integral Sliding Mode Control for an Underwater Robot With Unknown Disturbances and Uncertain Nonlinearities," IEEE Transactions on Industrial Electronics, vol. 64, no. 8, pp. 6785-6795, 2017.

[43] W. Xue and Y. Huang, "On performance analysis of ADRC for a class of MIMO lower-triangular nonlinear uncertain systems," ISA Transactions, vol. 53, no. 4, pp. 955-962, 2014. 
[44] Y. Pan, T. Sun, Y. Liu, and H. Yu, "Composite learning from adaptive backstepping neural network control," Neural Networks, vol. 95, pp. 134-142, 2017.

[45] Y. Pan and H. Yu, "Composite learning robot control with guaranteed parameter convergence," Automatica, vol. 89, pp. 398-406, 2017.

[46] X. Xiang, C. Liu, H. Su, and Q. Zhang, "On decentralized adaptive full-order sliding mode control of multiple UAVs," ISA Transactions, vol. 71, pp. 196-205, 2017.

[47] M. Krstić, I. Kanellakopoulos, and P. Kokotovic, Nonlinear and Adaptive Control Design, John Wiley \& Sons, 1995. 


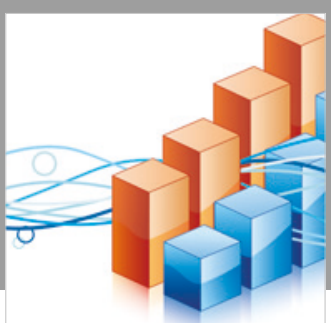

Advances in

Operations Research

\section{-n-m}
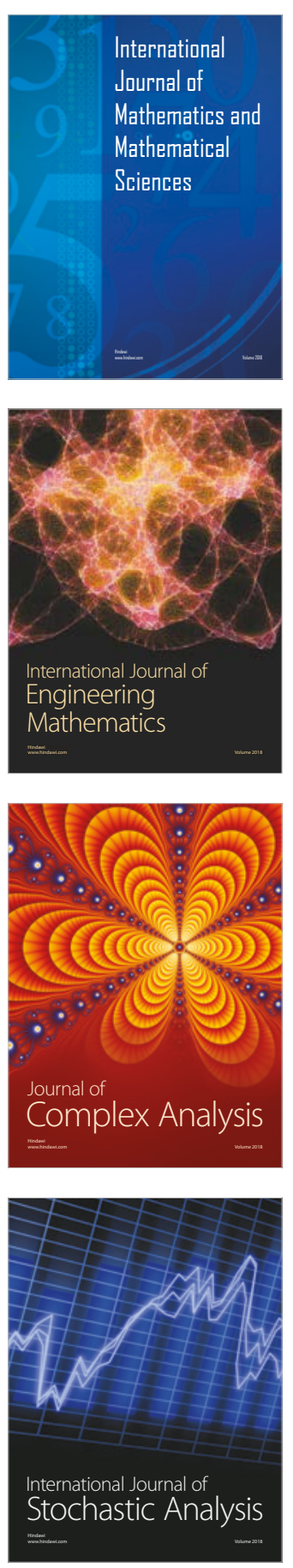
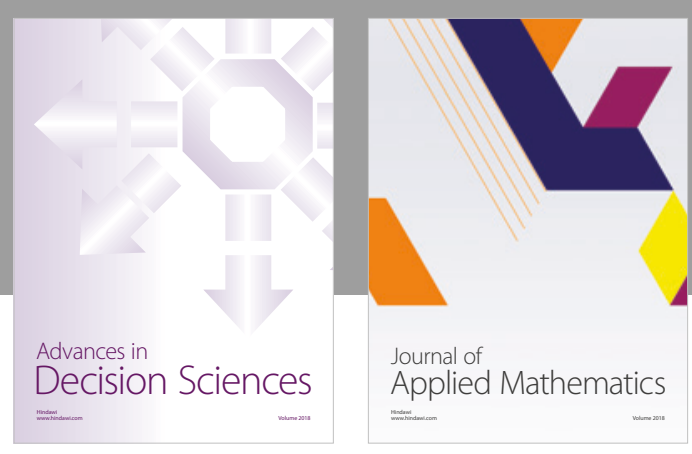

Journal of

Applied Mathematics
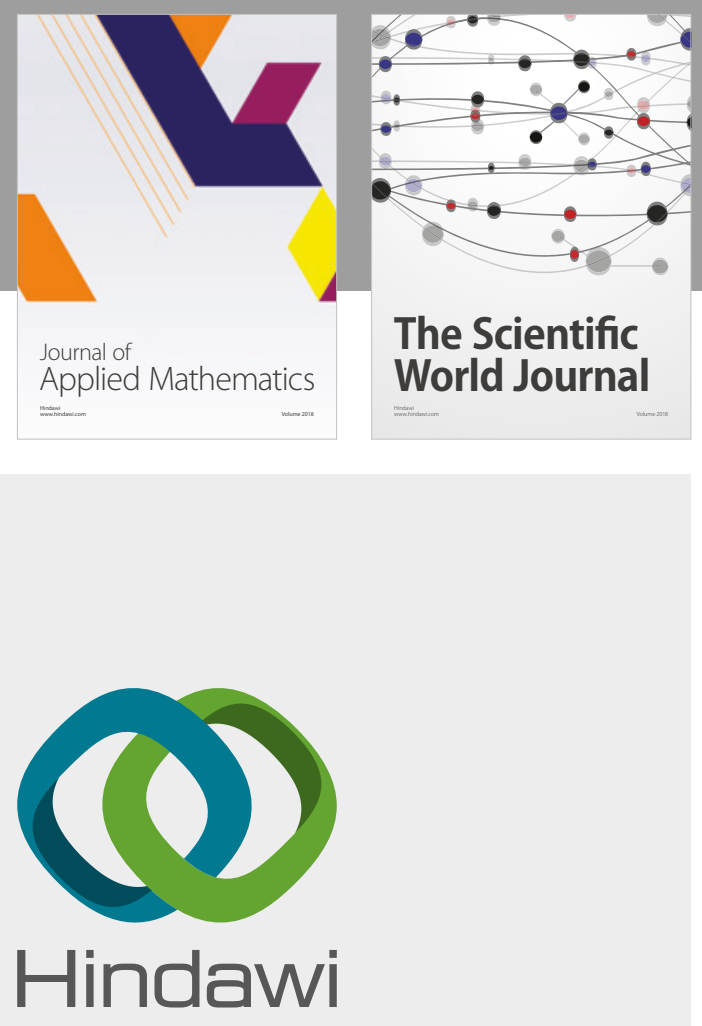

Submit your manuscripts at

www.hindawi.com

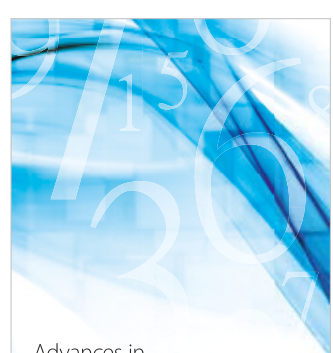

Advances in
Numerical Analysis
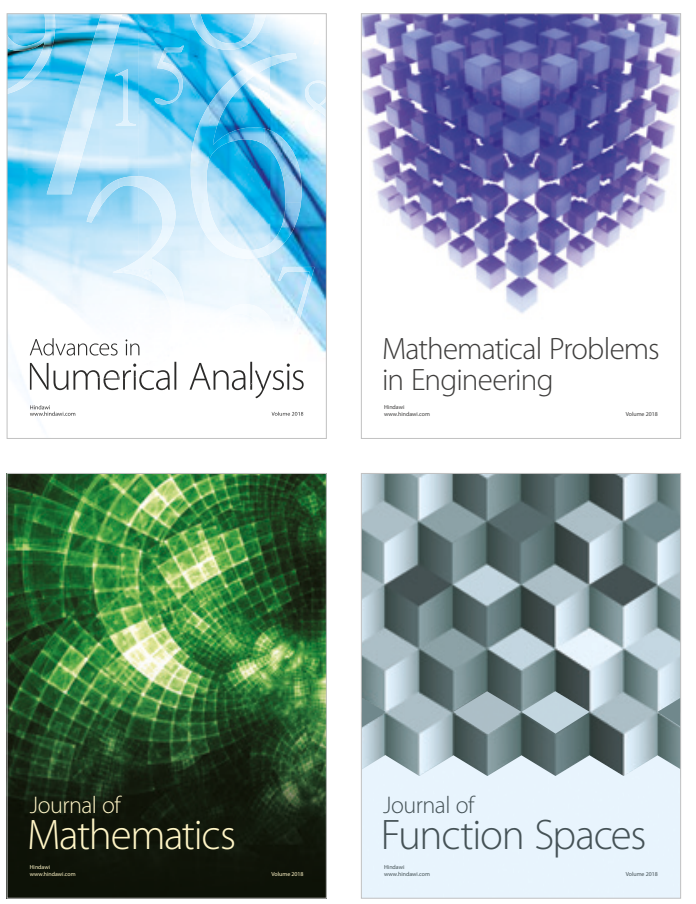

Mathematical Problems in Engineering

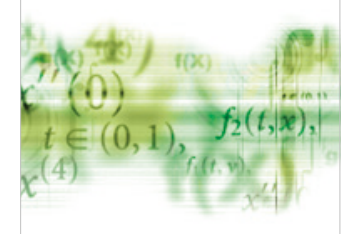

International Journal of

Differential Equations

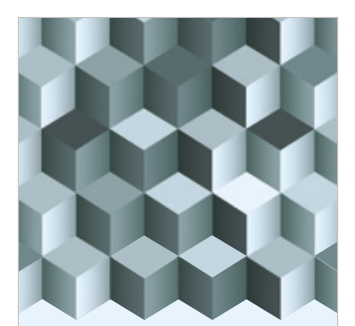

Journal of

Function Spaces
The Scientific

World Journal

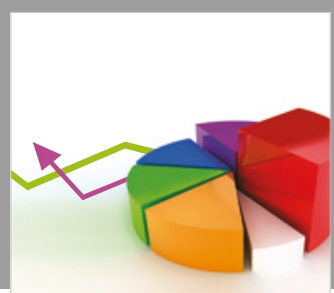

Journal of

Probability and Statistics
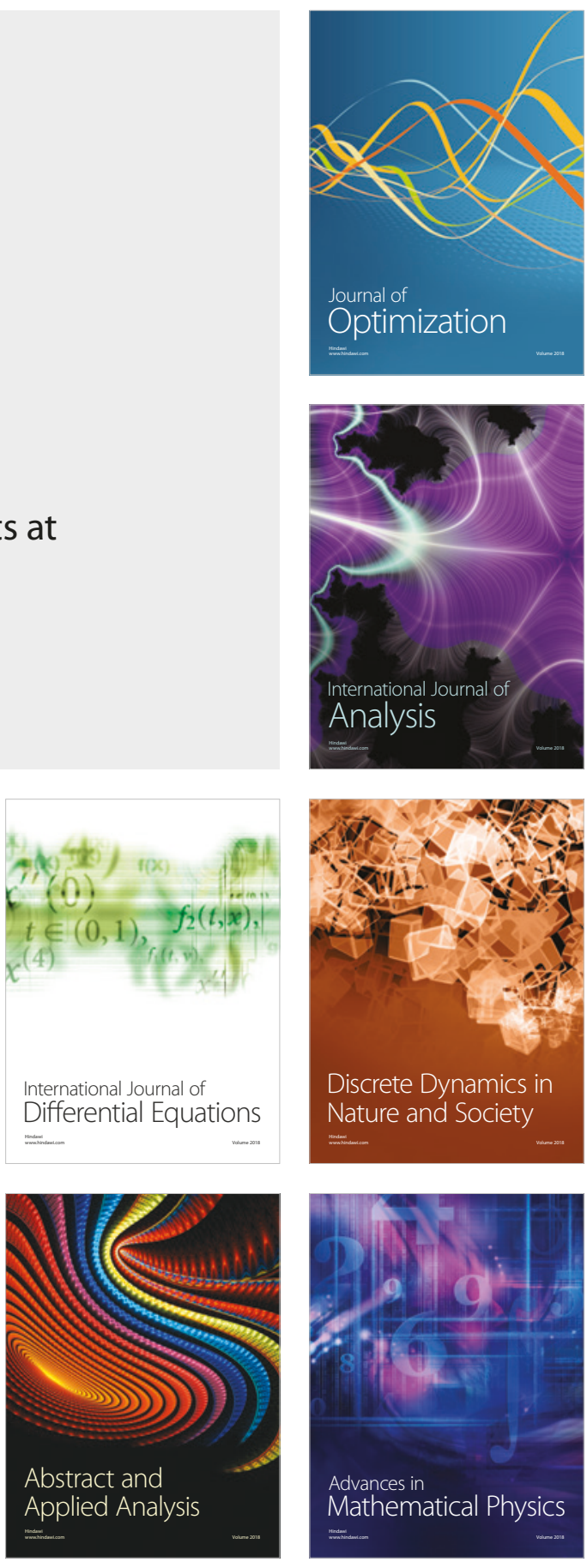